\title{
Fresh frozen plasma induced thrombocytopenia
}

\author{
Alexandra Drakaki ${ }^{1,2^{*}}$, Elizabeth Blanchard ${ }^{1,3^{*}}$ \\ ${ }^{1}$ Department of Medicine, Division of Hematology Oncology, Caritas St. Elizabeth Medical Center, Brighton, USA; \\ *Corresponding Authors: adrakaki@bidmc.harvard.edu, blanchard.eliz@gmail.com \\ ${ }^{2}$ Division of Hematology/Oncology, Department of Medicine, Beth Israel Deaconess Medical Center, Boston, USA \\ ${ }^{3}$ Southcoast Centers for Cancer Care, Fairhaven, USA
}

Received 15 March 2013; revised 19 April 2013; accepted 1 May 2013

Copyright (C 2013 Alexandra Drakaki, Elizabeth Blanchard. This is an open access article distributed under the Creative Commons Attribution License, which permits unrestricted use, distribution, and reproduction in any medium, provided the original work is properly cited.

\begin{abstract}
Transfusions of blood products are common in medical practice and can be lifesaving in certain situations. Potentially life threatening reactions could occur and physicians should be alerted. Here we describe a case of thrombocytopenia that was induced by transfusion of fresh frozen plasma (FFP). A 52 years old male presented to the emergency department after two episodes of hematochezia that resolved spontaneously. Since he was anticoagulated for atrial fibrillation he was given a unit of FFP to reverse a slightly elevated INR. Within 6 hours from the administration of the FFP he developed an acute decrease only in his platelet count. He was managed conservatively and his thrombocytopenia started resolving gradually. After excluding other causes the potential diagnosis was fresh frozen plasma induced thrombocytopenia. The pathophysiologic mechanism is postulated to be immune mediated by passive transfer of antibodyies from the donor to the recipient. The antibodies that are described in the literature are anti-HPA-1a and anti-CD-36. We reported the event to the American Red Cross. Interestingly a male was the donor of the plasma while in all cases in the literature the donors are females with a prior history of pregnancy. Therefore this is the first reported case of a male blood donor whose blood product caused immune mediated thrombocytopenia post transfusion.
\end{abstract}

Keywords: Thrombocytopenia; Fresh Frozen

Plasma; Blood Products

\section{INTRODUCTION}

Transfusion related adverse events are a known cause of morbidity. Fresh frozen plasma (FFP) is frequently used in patients with coagulopathy. There are 19 documented cases describing thrombocytopenia related to transfusion of FFP. The donors were exclusively females with a history of pregnancy; however this is the first described case of a patient transfused FFP from a male donor. The mechanism of thrombocytopenia is thought to be immune mediated by passive transfer of antibodies.

\section{CASE REPORT}

A 52 years old male presented to the emergency department for evaluation of rectal bleeding. His past medical history was significant for atrial fibrillation and a recent myocardial infarction. His medications included aspirin, clopidogrel, warfarin and metoprolol. He was hemodynamically stable and his physical examination was only positive for a $3 \mathrm{~mm}$ rectal fissure. Laboratory results were within normal limits except a slightly elevated prothrombin time and INR (Table 1). All of his medications were discontinued at that time even if his hematochezia had already been resolved. At that time the emergency medicine physician decided to transfuse the patient with fresh frozen plasma without any immediate adverse reaction. A complete blood count was repeated 6 hours later when an acute decrease of his platelet count was noted. The platelet count reached a nadir on day 4 . The other cell lines were unaffected and most importantly he never became anemic.

His peripheral blood smear revealed normal blood cell morphology, decreased number of platelets but no platelet clumping. A bone marrow biopsy was not performed since by the time we were consulted to review the case as hematology experts his platelet count started increasing (on day 5) and so we deferred any bone marrow biopsy or aspiration.

He underwent colonoscopy that revealed only internal hemorrhoids that probably explained his initial presentation. He never had any other episode of gastrointestinal 
Table 1. Laboratory values during hospitalization.

\begin{tabular}{|c|c|c|c|c|c|c|c|c|c|}
\hline & Day 1 & Day 2 & Day 3 & Day 4 & Day 5 & Day 6 & Day 7 & Day 8 & Day 9 \\
\hline $\begin{array}{l}\text { Prothrombin time } \\
\text { (normal range: } 11 \text { - } 13 \mathrm{sec} \text { ) }\end{array}$ & 15.3 & 14.8 & 14.2 & 14.1 & & & & & \\
\hline International normalized ratio & 1.2 & 1.2 & 1.1 & 1.1 & & & & & \\
\hline $\begin{array}{l}\text { Partial thromboplastin time } \\
\text { (normal range: } 25-35 \mathrm{sec} \text { ) }\end{array}$ & 25.4 & 23.6 & 23.8 & 23.4 & & & & & \\
\hline Fibrinogen (normal range: $150-350$ mg/dl) & 339 & NA & 292 & 275 & & & & & \\
\hline D-dimers (normal: $<0.5 \mu \mathrm{g} / \mathrm{ml}$ ) & 0.32 & NA & 0.24 & 0.34 & & & & & \\
\hline \multicolumn{10}{|l|}{$\begin{array}{l}\text { Platelet count } \\
\text { (normal range: } 150-450 \times 10^{9} / \mathrm{L} \text { ) }\end{array}$} \\
\hline $6 \mathrm{am}$ & & 69 & 35 & 30 & 37 & 37 & 48 & 67 & 80 \\
\hline $10 \mathrm{am}$ & 330 & & & & & & & & \\
\hline $4 \mathrm{pm}$ & 118 & 54 & 38 & & & & 60 & & \\
\hline
\end{tabular}

bleeding during his hospitalization even during the nadir of his platelet count.

He was discharged home on day 9. By that time there was an increasing trend of his platelet count (Table 1). Based on the literature the platelet recovery starts occurring after the first week as in our case. On his follow up outpatient visit his platelet count was completely normal.

We contacted the American Red Cross to report the case and to request the detection of the anti-platelet antibodies in the donor's blood. However what the American Red Cross considered important was to identify the donor and exclude him from the donor pool in order to prevent future events. Interestingly, a male was identified as the donor of the unit of the fresh frozen plasma.

\section{DISCUSSION}

The differential diagnosis of thrombocytopenia is broad. In our case thrombocytopenia due to clopidogrel or aspirin is unlikely since he was on those medications for a long time. Heparin induced thrombocytopenia (HIT type II) typically occurs 4 - 14 days after re-exposure [1]. He did receive heparin when he was recently hospitalized for a myocardial infarction however during this admission he was not exposed to heparin and the heaprin-PF4 antibody was tested and was undetectable. His clinical history along with his normal peripheral blood smear excluded sepsis, disseminated intravascular coagulation and thrombotic thrombocytopenic purpura (Table 1). The acuity of this presentation excluded immune thrombocytopenic purpura. The plasma transfusion was the most likely explanation which was supported by other case reports after reviewing the literature.

Fresh frozen plasma induced thrombocytopenia is immune mediated usually due to human platelet alloantigen-1a (HPA-1a) [2]. HPA-1a is carried by the subunit of the major fibrinogen receptor and is the main target for alloantibodies. Those are involved in post-transfusion purpura and fetal and neonatal alloimmune thrombocytopenia [3]. All previously reported cases of FFP induced thrombocytopenia have involved female donors with history of pregnancy [2], since it is more likely that they have developed alloantibodies during the perinatal period.

Another described antibody is the anti-CD36 which targets glycoprotein IIIb (or CD36) of the platelet surface and is also implicated in the immune mediated thrombocytopenia [4].

One case has also been described of passively transfused anti-CD36 isoantibody in a patient who received fresh frozen plasma from a donor who had Type I CD36 deficiency. In that case flow cytometric analysis identified the anti-CD36 isoantibody in the FFP and was considered the cause of the thrombocytopenia [5].

Others have proposed alternative mechanisms. In a case report of a patient with systemic lupus erythematosus who developed thrombocytopenia post fresh frozen plasma transfusion one proposed mechanism was the formation of microthrombi on the subendothelium as a result of endothelial cell activation [6].

\section{CONCLUSION}

An unexplained post transfusion thrombocytopenia should always be investigated for immune mediated causes even if it is not always possible to detect the implicated antibodies. Since this transfusion reaction is not that common there is not available literature supportive of the appropriate management in those cases. From our experience in a patient who is not actively bleeding a conservative approach is reasonable. However in a patient who is having signs and/or symptoms of bleeding due to the ongoing thrombocytopenia, a platelet transfusion could be considered [7]. Based on what is known so 
far it was interesting to find that this is the first reported case of a male donor of a blood product that induced immune mediated thrombocytopenia.

\section{REFERENCES}

[1] Bartholomew, J.R., Begelman, S.M. and Almahameed, A. (2005) Heparin-induced thrombocytopenia: Principles for early recognition and management. Cleveland Clinic Journal of Medicine, 72, 31-36. doi:10.3949/ccjm.72.Suppl 1.S31

[2] Pavenski, K., Webert, K.E. and Goldman, M. (2008) Consequences of transfusion of platelet antibody: A case report and literature review. Transfusion, 48, 1981-1989. doi:10.1111/j.1537-2995.2008.01796.x

[3] Kroll, H., Penke, G. and Santoso, S. (2005) Functional heterogeneity of alloantibodies against the human platelet antigen (HPA)-1a. Thrombosis and Haemostasis, 94, 12241229.

[4] Van Schravendijk, M.R., Handunnetti, S.M., Barnwell,
J.W. and Howard, R.J. (1992) Normal human erythrocytes express CD36, an adhesion molecule of monocytes, platelets, and endothelial cells. Blood, 80, 2105-2114.

[5] Morishita, K., Wakamoto, S., Miyazaki, T., Sato, S., Fujihara, M., Kaneko, S., Yasuda, H., Yamamoto, S., Azuma, H., Kato, T. and Ikeda, H. (2005) Life-threatening adverse reaction followed by thrombocytopenia after passive transfusion of fresh frozen plasma containing antiCD36 $\left(\mathrm{Nak}^{\mathrm{a}}\right)$ isoantibody. Transfusion, 45, 803-806. doi:10.1111/j.1537-2995.2005.04320.x

[6] Al-Shahi, R., Mason, J.C., Rao, R., Hurd, H., Thompson, E.M., Haskard, D.O. and Davies, K.A. (1997) Systemic lupus erythematosus, thrombocytopenia, microangiopathic haemolytic anemia and anti-CD36 antibodies. British Journal of Rheumatology, 36, 794-798. doi:10.1093/rheumatology/36.7.794

[7] Carr, J.M., Kruskall, M.S., Kaye, J.A. and Robinson, S.H. (1986) Efficacy of platelet transfusions in immune thrombocytopenia. American Journal of Medicine, 80, 10511054. doi:10.1016/0002-9343(86)90664-9 